\title{
Evaluation of Twelve Real-Time Reverse Transcriptase PCR Primer-Probe Sets for Detection of Pandemic Influenza A/H1N1 2009 Virus ${ }^{\nabla}$
}

\author{
Yaowu Yang, ${ }^{1,2} \dagger$ Fang Huang, ${ }^{3} \dagger$ Richard Gonzalez, ${ }^{2,4}$ Wei Wang, ${ }^{2}$ Guilan Lu, ${ }^{3}$ Yongjun Li, ${ }^{2,4}$ \\ Guy Vernet, ${ }^{4}$ Qi Jin, ${ }^{1 *}$ and Jianwei Wang ${ }^{1,2 *}$ \\ State Key Laboratory for Molecular Virology and Genetic Engineering, Institute of Pathogen Biology (IPB), Chinese Academy of \\ Medical Sciences and Peking Union Medical College (CAMS and PUMC), Beijing 100730, China ; Christophe Mérieux Laboratory, \\ IPB, CAMS-Fondation Mérieux, Beijing 100730, China ${ }^{2}$; Beijing Centre for Disease Control and Prevention, \\ Beijing 100013, China ${ }^{3}$; and Fondation Mérieux, 69365 Lyon, France ${ }^{4}$
}

Received 21 September 2010/Returned for modification 8 November 2010/Accepted 26 January 2011

\begin{abstract}
Real-time reverse transcriptase PCR (rRT-PCR) assays have greatly contributed to the detection, control, and prevention of the pandemic influenza A/H1N1 2009 virus. To improve the rRT-PCR assays for detection of pandemic influenza A/H1N1 2009 virus, we evaluated the sensitivity, specificity, and performance of 12 rRT-PCR primer-probe sets [SW (a) to SW (I)] using a panel of virus strains and clinical specimens. These primer-probe sets were derived from published work and designed for detecting the hemagglutinin (HA) or the neuraminidase (NA) gene of the pandemic influenza A/H1N1 2009 virus. A primer-probe set, SW (CDC), developed by the Centers for Disease Control and Prevention (U.S. CDC) to target the HA gene of pandemic influenza A/H1N1 2009 virus, was used as a referee method. Our results demonstrated that although all primer-probe sets in this study had as high as 98.4 to $100 \%$ in silico coverage, some of the primer-probe sets had better specificity, sensitivity, and amplification efficiency than others. Two primer-probe sets, SW (h) and SW (l), which target the NA gene of pandemic influenza A/H1N1 2009 virus, were highly sensitive $\left(1^{4}\right.$ copies/reaction), had high detection rates $(56 / 60, P=0.134$, and $59 / 60, P=1.000)$, and showed ideal specificity compared with SW (CDC). In addition, a cocktail of primer-probe sets targeted to the HA and NA genes displayed higher detection sensitivity than primer-probe sets targeting HA or NA alone, indicating that for practical applications, a combination of primer-probes targeting HA and NA genes is the best option for the detection of pandemic influenza A/H1N1 2009 virus.
\end{abstract}

The pandemic influenza A/H1N1 2009 virus, which was first identified in Mexico and the United States in April 2009 (5, 9), has rampaged globally for the past year. Although the World Health Organization (WHO) has declared that pandemic influenza A/H1N1 2009 virus is entering the postpandemic period (http://www.who.int/mediacentre/news/statements/2010 /h1n1_vpc_20100810/en/index.html), its future pattern remains to be determined. The pandemic influenza A/H1N1 2009 virus has been predicted to be the predominant influenza A virus causing seasonal influenza in winter/spring 2010-2011 (http: //www.cdc.gov/h1n1flu/qa.htm; http://www.ecdc.europa.eu/en /healthtopics/H1N1/Documents/1003_RA_forward_look_influenza .pdf). In addition, the recent identification of the re-sorting of this virus in pigs poses further, unknown risks to humans (25). Thus, detection, control, and prevention of an epidemic caused by pandemic influenza A/H1N1 2009 virus is still a global challenge, and an optimized detection method is needed in preparation for another possible outbreak.

Numerous assays for pandemic influenza A/H1N1 2009 virus have been reported, including virus isolation, enzyme-linked immunosorbent assay (ELISA), immunofluorescence analysis, and several kinds of molecular tests, such as conventional re-

\footnotetext{
* Corresponding author. Mailing address: No. 9 Dong Dan San Tiao, Dongcheng District, Beijing 100730, China. Phone and fax for J. Wang: 86-10-67828516. E-mail: wangjw28@163.com. Phone for Q. Jin: 86-10-67877732. Fax: 86-10-67877736. E-mail: zdsys@vip.sina.com.

$\dagger$ Y.Y. and F.H. contributed equally to this work.

${ }^{\nabla}$ Published ahead of print on 2 February 2011.
}

verse transcriptase PCR (cRT-PCR), real-time reverse transcriptase PCR (rRT-PCR), and sequence analysis (1, 3, 4, 11, 17, 18, 20, 22, 26). Among these, rRT-PCR has been widely used in various clinical and public health laboratories because of its unique advantages, including ease of performance, great accuracy, high sensitivity and specificity, fast turnaround time, high throughput capacity, and minimal carryover contamination (2, 23). In April 2009, the WHO released the Centers for Disease Control and Prevention (U.S. CDC) rRT-PCR protocol for the detection of pandemic influenza A/H1N1 2009 virus (http://www.who.int/csr/resources/publications/swineflu /CDCRealtimeRTPCR_SwineH1Assay-2009_20090430.pdf). This protocol has been widely used as the diagnostic standard in many countries and as the referee method in multiple studies $(6,14,18,24,27)$. Based on this, many rRT-PCR assays have been reported $(2,7,11,15,18,24,27,31-33)$. These assays used different primer and TaqMan probe sets to detect the hemagglutinin (HA) and/or the neuraminidase (NA) gene of pandemic influenza A/H1N1 2009 virus. As the primer-probe design plays a pivotal role in an rRT-PCR assay and the parameters of different primer-probe sets may differ $(28,30)$, careful evaluation of the sensitivities, specificities, and performances of different rRT-PCR primer-probe sets will help to improve the rRT-PCR assays for detection of pandemic influenza A/H1N1 2009 virus.

In this study, we used the SW (CDC) primer-probe set (SW H1 Forward, SW H1 Reverse, and SW H1 Probe in the U.S. CDC protocol) from the U.S. CDC's rRT-PCR protocol (http: //www.who.int/csr/resources/publications/swineflu/CDCRealtime 
RTPCR_SwineH1Assay-2009_20090430.pdf) to target the HA gene of pandemic influenza $\overline{\mathrm{A}} / \mathrm{H} 1 \mathrm{~N} 12009$ virus as a referee method. We evaluated the sensitivities, specificities, and performances of 12 primer-probe sets from rRT-PCR assays developed by different groups using a panel of respiratory virus strains and clinical samples. We demonstrate that these assays show different sensitivities, specificities, and performances for the detection of pandemic influenza A/H1N1 2009 virus.

\section{MATERIALS AND METHODS}

Virus stocks. A pandemic influenza A/H1N1 2009 virus strain (A/Beijing/01/ 2009 [GenBank accession numbers GQ183617 to GQ183624]) was isolated from Beijing, China. Two subtypes of seasonal influenza A virus strains, A/Puerto Rico/8/1934 (H1N1) and A/Jiangxi/424/2004 (H3N2), together with an influenza virus B/Shanghai/361/2002 strain, were provided by the Chinese National Influenza Center (CNIC). Human coronavirus OC43 (OC43) was provided by the Beijing Union Medical College Hospital. Adenovirus serotype 35 (Ad35, Holden strain) was purchased from the American Type Culture Collection (ATCC) (Manassas, VA). All of the viruses used in this study were grown in continuous cell lines (influenza A virus strains in MDCK cells, the influenza B virus strain and OC43 in LLC-MK2 cells, and Ad35 in HeLa cells).

Clinical-sample panels. An archived panel of 110 nasopharyngeal-swab specimens was used for the evaluation of rRT-PCR assays. Of those, 60 specimens collected between November 2009 and February 2010 were positive for pandemic influenza A/H1N1 2009 virus. These specimens were kindly provided by the Beijing Municipal Center for Disease Control and Prevention (Beijing CDC) and confirmed using U.S. CDC's rRT-PCR protocol for pandemic influenza A/H1N1 2009 virus. The other 50 specimens were randomly selected from specimens that were collected from adult patients diagnosed with susceptible viral acute respiratory tract infections at the Beijing Union Medical College Hospital (PUMCH) between June 2007 and August 2009. Multiplex or single PCR assays were used to test these specimens for common respiratory viruses, including influenza $\mathrm{A}, \mathrm{B}$, and $\mathrm{C}$ viruses; enterovirus; rhinovirus; parainfluenza virus types 1 to 4 ; metapneumovirus; coronavirus (OC43, 229E, NL-63, and HKU1); and adenovirus (29). Influenza virus HA/NA, including those of pandemic influenza A/H1N1 2009 virus, were subtyped using multiplex rRT-PCR assays as described previously (33). The PCR results were confirmed by sequencing. The 50 specimens included 15 positive for an $\mathrm{H} 3 \mathrm{~N} 2$ subtype, 16 positive for a seasonal H1N1 subtype, 2 positive for enterovirus, 2 positive for rhinovirus, 2 positive for parainfluenza virus, and 13 negative for the respiratory viruses we tested for as described above.

Viral RNA extraction. Viral RNA was extracted from clinical specimens or cultured viruses using the NucliSens easyMag apparatus (bioMérieux, Marcy L'Etoile, France) according to the manufacturer's protocol. The viral nucleic acids were stored at $-80^{\circ} \mathrm{C}$ prior to use.

Pandemic influenza A/H1N1 2009 virus rRT-PCR assays. Twelve rRT-PCR assays for pandemic influenza A/H1N1 2009 virus were selected from work published between July 2009 and July 2010. Details of these primer-probe sets are shown in Table 1. The thermocycling parameters for these primer-probe sets were based on original reports $(7,11,18,24,27,31-33)$. However, the number of PCR cycles was set at 45 , which was the highest cycle number used in the 12 evaluated assays (Table 1). We used the SW (CDC) primer-probe set from the U.S. CDC's rRT-PCR protocol to target the HA gene of pandemic influenza A/H1N1 2009 virus as a referee method. The thermocycling parameters for SW (CDC) were set according to the U.S. CDC's rRT-PCR protocol (http://www.who.int/csr/resources/publications /swineflu/CDCRealtimeRTPCR_SwineH1Assay-2009_20090430.pdf). For the sake of data analysis, all assays were performed using the same apparatus, a LightCycler 480 instrument (Roche Diagnostics, Mannheim, Germany), and the same one-step RT-PCR kit, the SuperScript III One-Step RT-PCR System with Platinum Taq DNA polymerase (Invitrogen, Carlsbad, CA). For all tests, $2 \mu \mathrm{l}$ of nucleic acid solution was added to $23 \mu \mathrm{l}$ of master mixture containing the following components at the indicated final concentrations: $1 \times$ reaction buffer, $1 \mu \mathrm{l}$ of SuperScript III RT/Platinum Taq Mix, $500 \mathrm{nM}$ each primer, and $300 \mathrm{nM}$ probe labeled at the $5^{\prime}$ end with a reporter dye, 6-carboxyfluorescein (FAM), and at the $3^{\prime}$ end with a nonfluorescent quencher, BHQ-1. Reverse transcription was performed for $30 \mathrm{~min}$ at $50^{\circ} \mathrm{C}$ and for $3 \mathrm{~min}$ at $95^{\circ} \mathrm{C}$ according to the manufacturer's instructions. The baseline fluorescence threshold for each analysis was manually adjusted based on the background fluorescence of the "no-template" control reaction using the baseline subtracted curve fit analysis mode in LightCycler 480 Software 1.5.0. For each analysis, a sample amplification curve exceeding baseline fluorescence with a corresponding cycle threshold $\left(C_{T}\right)$ value (not exceeding 36 in a 45 -cycle run) was considered positive. In addition, a $C_{T}$ value between 36 and 45 was considered weakly positive. An amplification curve lower than the baseline fluorescence was defined as negative.

In silico coverage of the primer-probe sets. A total of $1,848 \mathrm{HA}$ and 1,374 NA gene sequences of pandemic influenza A/H1N1 2009 virus were obtained from the Influenza Virus Resource (http://www.ncbi.nlm.nih.gov/genomes/FLU/) for in silico coverage analysis. In silico coverage of primer-probes was calculated using an in-house program, as previously described (33). A sequence was defined as a "hit" if there were two or fewer substitutions within the probe or if there were no substitutions within 5 bases from the $3^{\prime}$ end, one or fewer substitution within 10 bases from the $3^{\prime}$ end, or 3 or fewer substitutions within the primers (16). For degenerate primers or probes, an acceptable "hit" was defined by the presence of an accurate match between the sequence data consensus (made by BioEDIT 7.01 software) and its primers or probes.

Sensitivity, reproducibility, and specificity of rRT-PCR assays. The titers of the three known influenza A virus strains [A/Puerto Rico/8/1934 (H1N1), A/Jiangxi/424/2004 (H3N2), and A/Beijing/01/2009 (pandemic influenza A/H1N1 2009 virus)], B/Shanghai/361/2002 (influenza B virus), human coronavirus OC43, and $\mathrm{Ad} 35$ were measured by $50 \%$ tissue culture infective dose $\left(\mathrm{TCID}_{50}\right)$ and calculated by the Reed-Muench method (8). Copies of RNA extracts of the three known subtypes of influenza A virus strains and the other aforementioned viruses were quantified by SYBR green rRT-PCR $(10,33)$. The quantifications were done using standard curves made by serial dilutions of plasmids containing partial genes of influenza A and B virus NP, coronavirus RNA polymerase, and adenovirus hexon (data not shown). The limit of detection (LOD) and reproducibility analysis were determined using 10 -fold serial dilutions $\left(10^{7}\right.$ to 1 copies reaction or 4.0 to $4.0 \times 10^{-7} \mathrm{TCID}_{50} /$ reaction) of the quantified viral RNA extract of pandemic influenza A/H1N1 2009 virus. Stringent criteria for the LOD were adopted to evaluate the rRT-PCR assays: (i) to achieve the maximum $C_{T}$ values, (ii) to positively detect all three parallel wells (amplification curves exceeding the baseline fluorescence in a 45-cycle run), and (iii) to keep the coefficient of variation (CV) of the $C_{T}$ values less than $10 \%$. Viral RNA extracts were tested at high concentration and high $\operatorname{TCID}_{50}$ (Table 2) to determine the analytical specificity of the rRT-PCR assays. To further evaluate the specificities of different rRT-PCR assays in clinical testing, we used an additional 50 clinical nasopharyngeal-swab specimens, obtained from PUMCH as described above, for the cross-reaction assay (Table 3 ). All of the analytical assays were performed at least three times.

Statistical analysis. Statistical analysis for the rRT-PCR assays was performed using McNemar's chi-square test for matched pairs. A $P$ value of $\leq 0.05$ was considered significant.

\section{RESULTS}

In silico coverage of the primer-probe sets in this study. To evaluate the coverage of the 12 primer-probe sets derived from the published work for pandemic influenza A/H1N1 2009 virus strains, an in silico analysis was performed using an in-house program. The results showed that the coverage of these primerprobe sets ranged from $98.4 \%$ to $100 \%$ (Table 1), suggesting that all 12 primer-probe sets target the conserved regions of the HA or NA gene of pandemic influenza A/H1N1 2009 virus.

Specificities of rRT-PCR assays. To assess the specificities of the rRT-PCR assays, all of the sequences of the primerprobe sets were analyzed by BLAST analysis. The actual crossreactivity of the primer-probe sets was examined using a panel of virus strains and clinical specimens (Tables 2 and 3). Based on BLAST analysis, the SW (j) and SW (c) primer-probe sets, which target the HA gene of pandemic influenza A/H1N1 2009 virus, cross-react with the HA genes of swine H1N2 (such as the $\mathrm{A} /$ swine/Hainan/1/2005 strain) (Fig. 1A) and seasonal H1N1 (such as A/Puerto Rico/8/1934) (Fig. 1B), respectively. The other 10 primer-probe sets tested do not share obvious sequence homology, nor do they cross-react with other respiratory viruses (data not shown). Subsequent experiments also showed that the SW (c) set could detect the A/Puerto Rico/8/ 1934 strain (mean $C_{T}, 21.8$; standard deviation, 0.16; CV, 


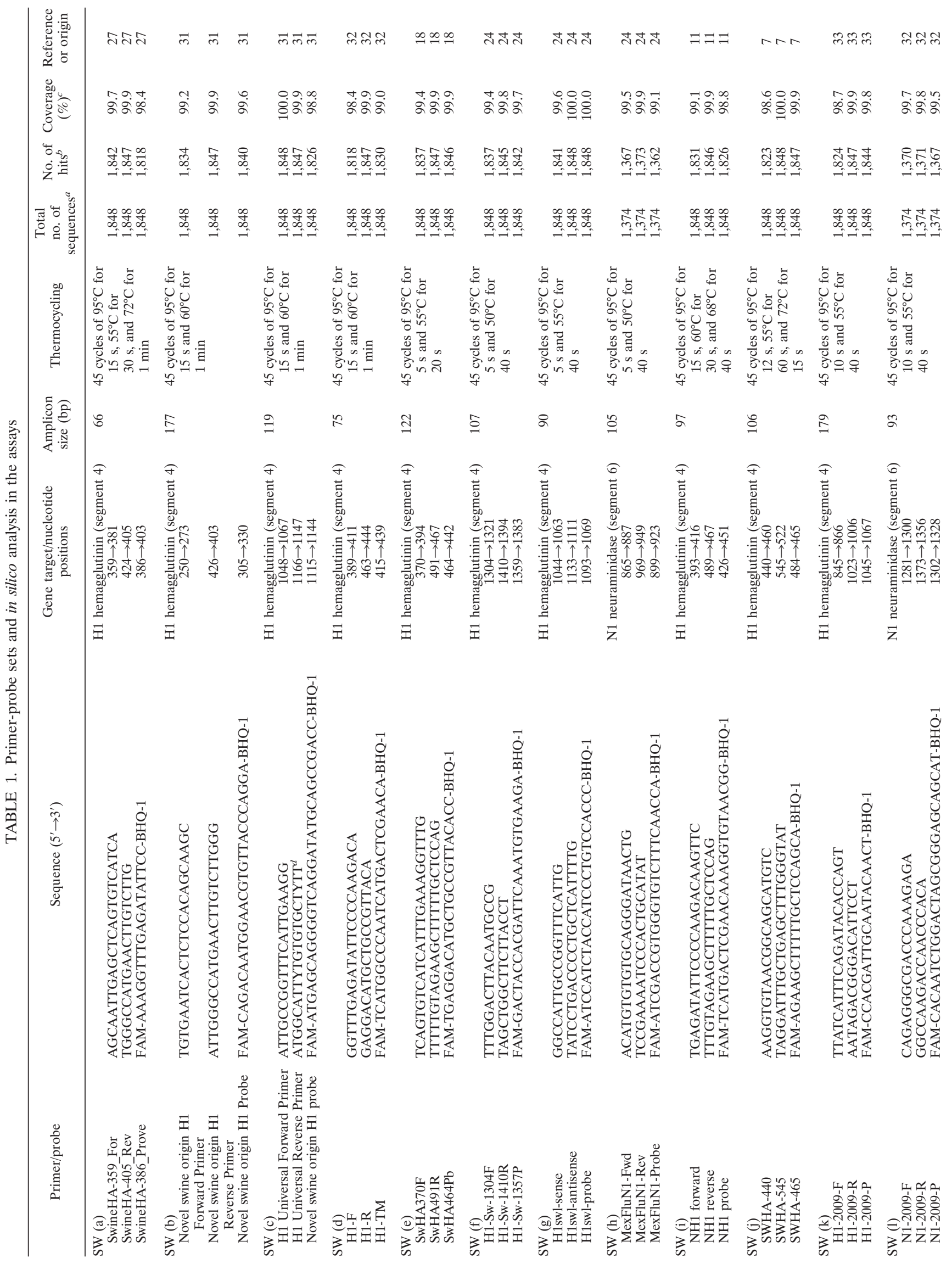


$0.73 \%$ ) (Table 2). The SW (j) set was not tested against the swine H1N2 subtype because the strain was unavailable. In addition, there were several other nonspecific, weakly positive reactions, such as SW (c) to Ad35 and SW (d) to influenza B virus and OC43 (Table 2). Other primer-probe sets, i.e., SW (a), SW (b), SW (e), SW (f), SW (h), SW (k), SW (l), and SW (CDC) (a primer-probe set in the U.S. CDC's rRT-PCR protocol to target $\mathrm{H} 1$ of pandemic influenza A/H1N1 2009 virus), did not obviously cross-react with other influenza virus subtypes or other respiratory viruses.

Sensitivities of rRT-PCR assays. The results of our LOD analysis showed that the 12 rRT-PCR assays detected the pandemic influenza A/H1N1 2009 virus strain A/Beijing/01/2009 with different efficiencies (Table 4). Based on the stringent conditions for the LOD, SW (d), SW (e), SW (g), and SW (k) detected pandemic influenza $\mathrm{A} / \mathrm{H} 1 \mathrm{~N} 12009$ virus most sensitively, with an LOD of $10^{3}$ copies/reaction, followed by SW (c), SW (h), SW (1), and SW (CDC), which had an LOD of $10^{4}$ copies/reaction.

Evaluation of rRT-PCR assays with clinical samples. We used a panel of 110 clinical samples to evaluate the performances of different rRT-PCR assays in clinical-sample detection (Table 5). Among the assays tested, five primer-probe sets [SW (c), (d), (e), (h), (i), and (l)] were not significantly different from SW (CDC) $\left(\chi^{2}\right.$ test; $P=0.480,1.000,0.480,0.134$, 1.000 , and 1.000 , respectively). Each set also had high detection rates for the 60 positive samples of pandemic influenza A/H1N1 2009 virus $(58,59,58,56,58$, and 59, respectively).

Several sets nonspecifically detected other strains. SW (d) nonspecifically detected H3N2 (2/15), SW (g) detected seasonal H1N1 (11/16) and parainfluenza virus (1/2), and SW (i) detected seasonal H1N1 (1/16) (Tables 3 and 5). In addition, SW $(\mathrm{g})$ reacted weakly with parainfluenza virus $(1 / 2)$ and one negative specimen (Table 3 ).

Based on the clinical-sample testing results (Tables 3 and 5) and the specificity and sensitivity analyses (Tables 2 and 4 and Fig. 1), SW (e), SW (h), and SW (l) were the ideal primerprobe sets. In addition, the combination of SW (e) (HA targeted) and SW (h) (NA targeted) increased the detection rate to $59 / 60$ ( $\chi^{2}$ test; $P=1.000$ ), and the combination of SW (e) (HA targeted) and SW (l) (NA targeted) increased the detection rate to $60 / 60\left(\chi^{2}\right.$ test; $\left.P=1.000\right)$ (Table 5), indicating that a combination of primer-probe sets more effectively detects target viruses than individual primer-probe sets alone.

\section{DISCUSSION}

Although rRT-PCR assays are more sensitive than conventional reverse transcriptase PCR assays (21) and rapid antigenbased tests $(12,13)$, each rRT-PCR assay may be different in sensitivity, specificity, and performance for the detection of pandemic influenza A/H1N1/2009 virus infection due to different design criteria and target genes. In this study, we evaluated 12 primer-probe sets developed by different groups for the detection of pandemic influenza A/H1N1 2009 virus. Our results demonstrated that although all primer-probe sets in this study had as high as 98.4 to $100 \%$ in silico coverage (Table 1), some of the primer-probe sets have better specificity, sensitivity, and amplification efficiency than others.

Based on the results of the specificity analysis in this study, 
TABLE 2. Analysis of the specificities of the different rRT-PCR assays using a panel of virus strains

\begin{tabular}{|c|c|c|c|c|c|c|c|c|c|c|c|c|c|c|c|c|}
\hline Virus strain & Subtype & $\begin{array}{c}\mathrm{TCID}_{50} / \\
\mathrm{ml}\end{array}$ & $\begin{array}{l}\text { No. of copies/ } \\
\text { reaction }\end{array}$ & SW (a) & SW (b) & $\mathrm{SW}(\mathrm{c})$ & SW (d) & SW (e) & SW (f) & SW (g) & SW (h) & SW (i) & SW (j) & SW (k) & SW (l) & $\begin{array}{c}\mathrm{SW} \\
(\mathrm{CDC})^{a}\end{array}$ \\
\hline $\mathrm{A} / \mathrm{PR} / 8 / 1934$ & H1N1 & $9.4 \times 10^{4}$ & $1 \times 10^{7}$ & $-^{d}$ & - & $++^{b}$ & - & - & - & - & - & - & - & - & - & - \\
\hline A/Beijing/01/2009 & A/H1N1 2009 & $1.6 \times 10^{3}$ & $1 \times 10^{8}$ & + & + & + & + & + & + & + & + & + & + & + & + & + \\
\hline A/Jiangxi/242/2004 & $\mathrm{H} 3 \mathrm{~N} 2$ & $4.6 \times 10^{4}$ & $1 \times 10^{8}$ & - & - & - & - & - & - & - & - & - & - & - & - & - \\
\hline $\mathrm{B} / \mathrm{SH} / 361 / 2002$ & Influenza B & $1.4 \times 10^{4}$ & $4 \times 10^{4}$ & - & - & - & $\pm^{c}$ & - & - & - & - & - & - & - & - & - \\
\hline $\mathrm{OC} 43$ & & $1.4 \times 10^{6}$ & $2 \times 10^{6}$ & - & - & - & \pm & - & - & - & - & - & - & - & - & - \\
\hline Adenovirus Holden & Serotype 35 & $1.4 \times 10^{7}$ & $2 \times 10^{9}$ & - & - & \pm & - & - & - & - & - & - & - & - & - & - \\
\hline
\end{tabular}

${ }^{a}$ The SW (CDC) primer-probe set (SW H1 Forward, SW H1 Reverse, and SW H1 Probe in U.S. CDC protocol) was from U.S. CDC's rRT-PCR assay, which is recommended by the World Health Organization and has been used for clinical screening as the diagnostic gold standard for pandemic influenza A/H1N1 2009 virus in many countries and regions.

${ }^{b}$ The mean $C_{T}$ s, standard deviations, and CVs for " + " were all 8.8 to $21.8,0.02$ to 2.07 , and 0.20 to $2.97 \%$, respectively.

${ }^{c} \pm, C_{T}$ s at 36.0 to 45.0 .

$d_{-}$, negative results in which the amplification curve values were lower than the baseline fluorescence values.

the SW (j) (7) and SW (c) (31) sets are highly homologous to the HA genes of swine H1N2 (Fig. 1A) and to those of the human H1N1 strains (Fig. 1B and Table 2), respectively. The high identity between SW (j) and swine H1N2 is consistent with the notion that the pandemic influenza A/H1N1 2009 virus was a reassortant (swine $\mathrm{H} 1 \mathrm{~N} 2 \times$ Eurasian swine $\mathrm{H} 1 \mathrm{~N} 1$ ) that obtained its HA gene from swine H1N2 (19). We examined the cross-reactivities of primer-probe sets using a panel of virus strains and clinical specimens and found several primerprobe sets with high specificity [such as SW (a) (27), SW (h) (24), and SW (1) (32)]. However, considering the numerous HA and NA subtypes and the complicated evolutionary relationships between influenza A virus subtypes, the specificities of the primer-probe sets need to be further evaluated.

As demonstrated in this report, it is often difficult to achieve both high sensitivity and high specificity for viral nucleic acid detection. The primer-probe sets SW (d) (32), SW (e) (18), SW (g) (24), and SW (i) (11) are highly sensitive $\left(10^{3}\right.$ copies/ reaction) but react nonspecifically with other subtypes or viruses (Tables 2, 3, and 5). Given this, the two NA gene-targeted sets, SW (h) and SW (1), and the HA gene-targeted set SW (e) appear to be the ideal primer-probe sets, because they had relatively high sensitivity $\left[10^{4}\right.$ copies/reaction for SW (h) and SW (1) and $10^{3}$ copies/reaction for SW (e)] and detection rates $[56 / 60, P=0.134$, for $\mathrm{SW}(\mathrm{h}) ; 59 / 60, P=1.000$, for $\mathrm{SW}$ (1); and $58 / 60, P=0.480$, for SW (e)], as well as ideal specificity (Tables 2, 3, and 5). Surprisingly, our results showed that the SW (f) (21) set had a relatively low sensitivity and detection rate (Tables 4 and 5) for pandemic influenza A/H1N1 2009 virus, although the optimized annealing temperature, $50^{\circ} \mathrm{C}$, was selected from a series of temperatures. This may be attributed to the lower melting temperature $\left(T_{m}\right)$ values of the primers and probe. The $T_{m}$ values of the forward primer, reverse primer, and probe of the $\mathrm{SW}$ (f) set were $47^{\circ} \mathrm{C}, 39^{\circ} \mathrm{C}$, and $54^{\circ} \mathrm{C}$, respectively, which are lower than the $T_{m}$ values recommended in the guidelines for designing primer-probe sets for quantitative assays (primers, 58 to $60^{\circ} \mathrm{C}$; probes, 68 to $70^{\circ} \mathrm{C}$ ) (http://www.uic.edu/depts/rrc/cgf/).

To keep the PCR parameters consistent with the original parameters and to derive accurate evaluation results, we did not change any parameters except the number of PCR cycles. Because the number of PCR cycles differed among the 12 assays compared in our study and the different numbers of rRT-PCR cycles may affect the comparisons of sensitivity and specificity, we set the number of PCR cycles to 45 in all assays. This number corresponds to the largest cycle number used in the 12 evaluated assays. The adjustment of cycle numbers did not affect the sensitivities and specificities of the primer-probe sets based on our comparisons (data not shown).

Compared with the U.S. CDC's rRT-PCR protocol for pandemic influenza A/H1N1 2009 virus, the SW (a), (b), (f), (j), and $(\mathrm{k})$ primer-probe sets are less sensitive and less specific. Considering that there is no preferred standard method for evaluating rRT-PCR assays used to detect pandemic influenza A/H1N1 2009 virus, these assays should be further evaluated

TABLE 3. Analysis of the specificities of the different rRT-PCR assays using a panel of clinical specimens positive for other viruses

\begin{tabular}{|c|c|c|c|c|c|c|c|c|c|c|c|c|c|c|}
\hline \multirow{2}{*}{$\begin{array}{l}\text { Clinical specimen } \\
\text { type/subtype }\end{array}$} & \multirow{2}{*}{$\begin{array}{l}\text { No. of } \\
\text { samples } \\
\text { tested }\end{array}$} & \multicolumn{13}{|c|}{ No. positive } \\
\hline & & SW (a) & SW (b) & SW (c) & SW (d) & SW (e) & SW (f) & SW (g) & SW (h) & SW (i) & SW (j) & SW (k) & SW (l) & $\begin{array}{c}\mathrm{SW} \\
(\mathrm{CDC})^{a}\end{array}$ \\
\hline Seasonal H3N2 & 15 & 0 & 0 & 0 & $2(+)^{b}$ & 0 & 0 & 0 & 0 & 0 & 0 & 0 & 0 & 0 \\
\hline Seasonal H1N1 & 16 & 0 & 0 & 0 & 0 & 0 & 0 & $11(+)$ & 0 & $1(+)$ & 0 & 0 & 0 & 0 \\
\hline Enterovirus & 2 & 0 & 0 & 0 & 0 & 0 & 0 & 0 & 0 & 0 & 0 & 0 & 0 & 0 \\
\hline Parainfluenza virus & 2 & 0 & 0 & 0 & 0 & 0 & 0 & $1( \pm)^{c}$ & 0 & 0 & 0 & 0 & 0 & 0 \\
\hline Rhinovirus & 2 & 0 & 0 & 0 & 0 & 0 & 0 & 0 & 0 & 0 & 0 & 0 & 0 & 0 \\
\hline Negative samples ${ }^{d}$ & 13 & 0 & 0 & 0 & 0 & 0 & 0 & $1( \pm)$ & 0 & 0 & 0 & 0 & 0 & 0 \\
\hline
\end{tabular}

${ }^{a}$ The SW (CDC) primer-probe set (SW H1 Forward, SW H1 Reverse, and SW H1 Probe in U.S. CDC protocol) was from U.S. CDC's rRT-PCR assay, which is recommended by the World Health Organization and has been used for clinical screening as the diagnostic gold standard for pandemic influenza A/H1N1 2009 virus in many countries and regions.

$b+, C_{T}$ s were less than 36.0

${ }^{c} \pm, C_{T}$ s were 36.0 to 45.0 .

${ }^{d}$ Negative samples were negative for the common respiratory viruses, such as influenza virus, enterovirus, rhinovirus, parainfluenza virus, metapneumovirus, coronavirus. and adenovirus. 


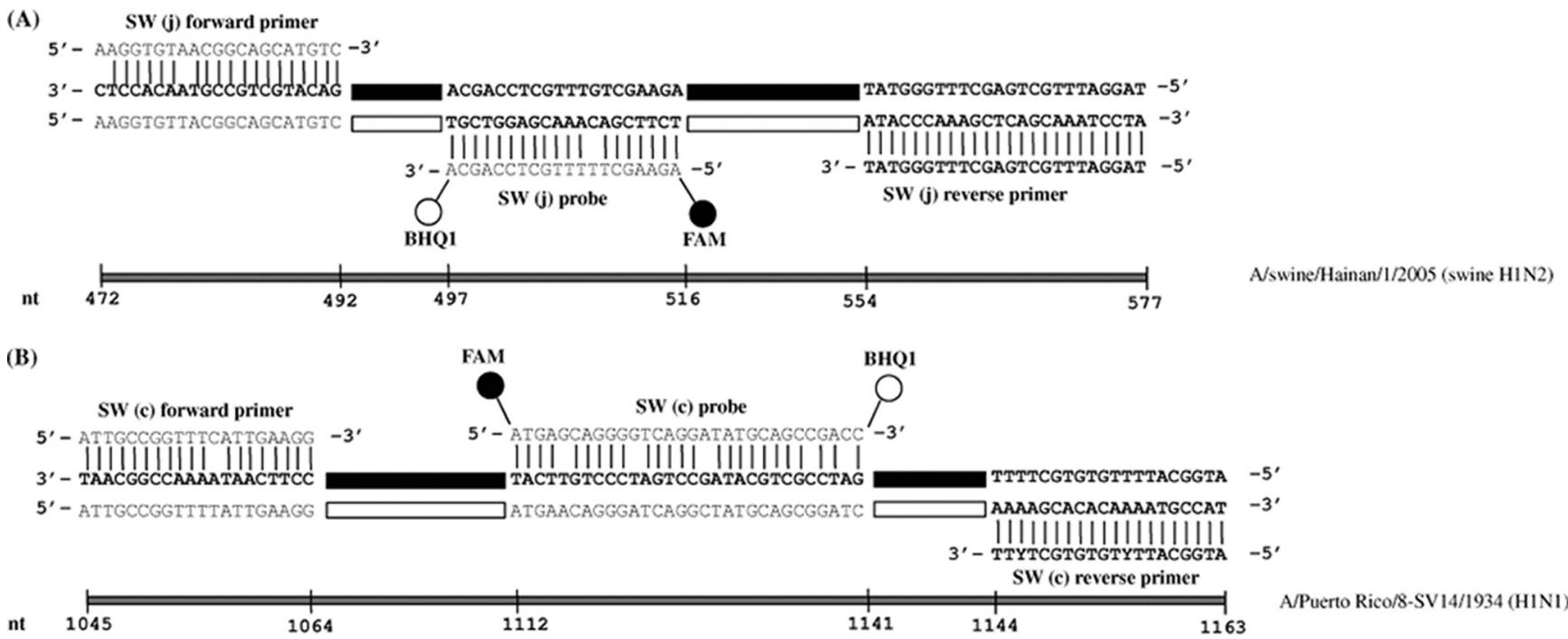

FIG. 1. Sequence alignment of the primer-probe sets with nonspecific viruses. (A) Pairwise alignment of nucleotides between the SW (j) (7) primer-probe set and the HA gene of a swine H1N2 strain (A/swine/Hainan/1/2005 [GenBank accession number EF556203]). (B) Pairwise alignment of nucleotides between the SW (c) (31) primer-probe set and the HA gene of a human H1N1 strain (A/Puerto Rico/8/1934 [GenBank accession number EF556203]).

by a third-party referee in the future. Our preliminary conclusion may provide a basis for such future studies.

Due to limitations of primer-probe sets targeting a single gene and leading to false-negative diagnosis when sequence variations exist in the primer- and/or probe-targeting region, we evaluated two primer-probe sets to target the HA and NA genes simultaneously in order to increase the sensitivity of the rRT-PCR assay for detecting pandemic influenza $\mathrm{A} / \mathrm{H} 1 \mathrm{~N} 1$ 2009 virus. Our results show that from the standpoint of sensitivity and specificity, the cocktail of SW(e) (HA) and SW(h) (NA) or SW (e) (HA) and SW (1) (NA) is a recommended improvement over the single set of primers for the detection of

TABLE 4. Analysis of the LODs of the different rRT-PCR assays

\begin{tabular}{lcccccc}
\hline & \multicolumn{2}{c}{ LOD $^{a}$} & & \multicolumn{3}{c}{$C_{T}$} \\
\cline { 2 - 3 } \cline { 6 - 7 } Assay & $\begin{array}{c}\text { No. of copies/ } \\
\text { reaction }\end{array}$ & TCID $_{50}$ /reaction & & Mean & SD & CV (\%) \\
& $1.0 \times 10^{5}$ & $4.0 \times 10^{-2}$ & & 28.27 & 0.78 & 2.76 \\
SW (a) & $1.0 \times 10^{6}$ & $4.0 \times 10^{-1}$ & & 23.12 & 0.43 & 1.87 \\
SW (b) & $1.0 \times 10^{4}$ & $4.0 \times 10^{-3}$ & & 31.98 & 0.08 & 0.26 \\
SW (c) & $1.0 \times 10^{3}$ & $4.0 \times 10^{-4}$ & & 33.31 & 0.31 & 0.93 \\
SW (d) & $1.0 \times 10^{3}$ & $4.0 \times 10^{-4}$ & & 35.44 & 1.23 & 3.48 \\
SW (e) & $1.0 \times 10^{7}$ & 4.0 & & 33.28 & 1.86 & 5.59 \\
SW (f) & $1.0 \times 10^{3}$ & $4.0 \times 10^{-4}$ & & 31.89 & 0.5 & 1.56 \\
SW (g) & $1.0 \times 10^{4}$ & $4.0 \times 10^{-3}$ & & 29.51 & 0.08 & 0.26 \\
SW (h) & $1.0 \times 10^{4}$ & $4.0 \times 10^{-3}$ & & 29.26 & 0.51 & 1.74 \\
SW (i) & $1.0 \times 10^{5}$ & $4.0 \times 10^{-2}$ & & 26.9 & 0.08 & 0.3 \\
SW (j) & $1.0 \times 10^{3}$ & $4.0 \times 10^{-4}$ & & 33.64 & 0.66 & 1.96 \\
SW (k) & $1.0 \times 10^{4}$ & $4.0 \times 10^{-3}$ & & 33.45 & 0.61 & 1.82 \\
SW (l) & $1.0 \times 10^{4}$ & $4.0 \times 10^{-3}$ & & 32.37 & 0.42 & 1.31 \\
SW (CDC) $b$ & $1.0 \times 1.0 \times 13$ &
\end{tabular}

${ }^{a}$ The sufficient conditions of LODs were $3 / 3$ amplification curves exceeding the baseline fluorescence in a 45 -cycle run and $C_{T}$ CVs of less than $10 \%$.

${ }^{b}$ The SW (CDC) primer-probe set (SW H1 Forward, SW H1 Reverse, and SW H1 Probe in U.S. CDC protocol) was from U.S. CDC's rRT-PCR assay, which is recommended by the World Health Organization and has been used for clinical screening as the diagnostic gold standard for pandemic influenza A/H1N1 2009 virus in many countries and regions.
TABLE 5. Comparisons of the different rRT-PCR assays for the detection of pandemic influenza A/H1N1 2009 virus

\begin{tabular}{|c|c|c|c|c|c|}
\hline \multirow[t]{2}{*}{ rRT-PCR assay } & \multirow[t]{2}{*}{ Result } & \multicolumn{2}{|c|}{$\begin{array}{l}\text { No. by SW } \\
(\mathrm{CDC})^{a}\end{array}$} & \multirow{2}{*}{$\chi^{2}$} & \multirow{2}{*}{$P^{b}$} \\
\hline & & + & - & & \\
\hline \multirow[t]{2}{*}{ SW (a) } & + & 48 & 0 & 10.08 & 0.002 \\
\hline & - & 12 & 50 & & \\
\hline \multirow[t]{2}{*}{ SW (b) } & + & 44 & 0 & 14.06 & 0.000 \\
\hline & - & 16 & 50 & & \\
\hline \multirow[t]{2}{*}{ SW (c) } & + & 58 & 0 & 0.50 & 0.480 \\
\hline & - & 2 & 50 & & \\
\hline \multirow[t]{2}{*}{ SW (d) } & + & 59 & 2 & 0.00 & 1.000 \\
\hline & - & 1 & 48 & & \\
\hline \multirow[t]{2}{*}{ SW (e) } & + & 58 & 0 & 0.50 & 0.480 \\
\hline & - & 2 & 50 & & \\
\hline \multirow[t]{2}{*}{ SW (f) } & + & 36 & 0 & 22.04 & 0.000 \\
\hline & - & 24 & 50 & & \\
\hline \multirow[t]{2}{*}{ SW (g) } & + & 58 & 12 & 5.79 & 0.016 \\
\hline & - & 2 & 38 & & \\
\hline \multirow[t]{2}{*}{ SW (h) } & + & 56 & 0 & 2.25 & 0.134 \\
\hline & - & 4 & 50 & & \\
\hline \multirow[t]{2}{*}{ SW (i) } & + & 58 & 1 & 0.00 & 1.000 \\
\hline & - & 2 & 49 & & \\
\hline \multirow[t]{2}{*}{ SW (j) } & + & 50 & 0 & 8.10 & 0.004 \\
\hline & - & 10 & 50 & & \\
\hline \multirow[t]{2}{*}{$\mathrm{SW}(\mathrm{k})$} & + & 51 & 0 & 7.11 & 0.008 \\
\hline & - & 9 & 50 & & \\
\hline \multirow[t]{2}{*}{ SW (1) } & + & 59 & 0 & 0.00 & 1.000 \\
\hline & - & 1 & 50 & & \\
\hline \multirow[t]{2}{*}{$\mathrm{SW}(\mathrm{e})+\mathrm{SW}(\mathrm{h})$} & + & 59 & 0 & 0.00 & 1.000 \\
\hline & - & 1 & 50 & & \\
\hline \multirow[t]{2}{*}{$\mathrm{SW}(\mathrm{e})+\mathrm{SW}(1)$} & + & 60 & 0 & 0.00 & 1.000 \\
\hline & - & 0 & 50 & & \\
\hline
\end{tabular}

${ }^{a}$ The SW (CDC) primer-probe set (SW H1 Forward, SW H1 Reverse, and SW H1 Probe in U.S. CDC protocol) was from U.S. CDC's rRT-PCR assay, which is recommended by the World Health Organization and has been used for clinical screening as the diagnostic gold standard for pandemic influenza A/H1N1 2009 virus in many countries and regions.

${ }^{b}$ Statistical significance was determined via McNemar's chi-square test for matched pairs. A $P$ value of $\leq 0.05$ was considered significant. Boldface indicates values $>0.05$, representing no statistical significance for the results of comparisons of the evaluated RT-PCR to the U.S. CDC's rRT-PCR assay. 
HA or NA alone. The detection rates of the combination of SW (e) (HA targeted) and SW (h) (NA targeted) and the combination of SW (e) (HA targeted) and SW (1) (NA targeted) were $59 / 60(P=1.000)$ and $60 / 60(P=1.000)$, respectively, while those of SW(e), SW (h), and SW (l) alone were $58 / 60,56 / 60$, and 59/60, respectively.

In summary, we evaluated 12 rRT-PCR primer-probe sets for the detection of pandemic influenza A/H1N1 2009 virus. The disparities in specificity, sensitivity, and amplification were demonstrated by tests using virus strains and clinical samples. Our study provides useful information on the optimization of rRT-PCR for the detection of pandemic influenza A/H1N1 2009 virus showing that a combination of primers and probes targeting the HA and NA genes may be the best option for the detection of pandemic influenza A/H1N1 2009 virus.

\section{ACKNOWLEDGMENTS}

This work was supported in part by the National Basic Research Program of China (2010CB534003), the International Science and Technology Cooperation Program of China (2010 DFB33270), and an intramural grant from the Institute of Pathogen Biology, Chinese Academy of Medical Sciences (2006IPB05).

\section{REFERENCES}

1. Boggild, A. K., and A. J. McGeer. 2010. Laboratory diagnosis of 2009 H1N1 influenza A virus. Crit. Care Med. 38:e38-e42.

2. Butot, S., et al. 2010. Evaluation of various real-time RT-PCR assays for the detection and quantitation of human norovirus. J. Virol. Methods 167:90-94.

3. Carr, M. J., et al. 2009. Development of a real-time RT-PCR for the detection of swine-lineage influenza A (H1N1) virus infections. J. Clin. Virol. 45:196-199.

4. Centers For Disease Control And Prevention. 10 August 2009. Interim guidance for the detection of novel influenza A virus using rapid influenza diagnostic tests. http://www.cdc.gov/h1n1flu/guidance/rapid testing.htm.

5. Centers For Disease Control and Prevention. 2009. Swine influenza A (H1N1) infection in two children-Southern California, March-April 2009. MMWR Morb. Mortal. Wkly. Rep. 58:400-402.

6. Cheung, T. K., et al. 2010. Evaluation of novel H1N1-specific primer-probe sets using commercial RT-PCR mixtures and a premixed reaction stored in a lyophilized format. J. Virol. Methods 165:302-304.

7. Chidlow, G., et al. 2010. Duplex real-time reverse transcriptase PCR assays for rapid detection and identification of pandemic (H1N1) 2009 and seasonal influenza A/H1, A/H3, and B viruses. J. Clin. Microbiol. 48:862-866.

8. Cunningham, C. H. 1973. Quantal and enumerative titration of virus in cell cultures, p. 527-532. In P. F. Kruse, Jr., and M. K. Patterson, Jr. (ed.), Tissue culture: methods and application. Academic Press, Inc., New York, NY.

9. Dawood, F. S., et al. 2009. Emergence of a novel swine-origin influenza A (H1N1) virus in humans. N. Engl. J. Med. 360:2605-2615.

10. Dhanasekaran, S., T. M. Doherty, and J. Kenneth. 2010. Comparison of different standards for real-time PCR-based absolute quantification. J. Immunol. Methods 354:34-39.

11. Dong, H., et al. 2010. Detection of human novel influenza A (H1N1) viruses using multi-fluorescent real-time RT-PCR. Virus Res. 147:85-90.
12. Drexler, J. F., et al. 2009. Poor clinical sensitivity of rapid antigen test for influenza A pandemic (H1N1) 2009 virus. Emerg. Infect. Dis. 15:1662-1664.

13. Faix, D. J., S. S. Sherman, and S. H. Waterman. 2009. Rapid-test sensitivity for novel swine-origin influenza A (H1N1) virus in humans. N. Engl. J. Med. 361:728-729.

14. Ginocchio, C. C., et al. 2009. Evaluation of multiple test methods for the detection of the novel 2009 influenza A (H1N1) during the New York City outbreak. J. Clin. Virol. 45:191-195.

15. Hall, R. J., M. Peacey, Q. S. Huang, and P. E. Carter. 2009. Rapid method to support diagnosis of swine origin influenza virus infection by sequencing of real-time PCR amplicons from diagnostic assays. J. Clin. Microbiol. 47: 3053-3054.

16. He, J., et al. 2009. Rapid multiplex reverse transcription-PCR typing of influenza A and B virus, and subtyping of influenza A virus into $\mathrm{H} 1,2,3,5$, 7, 9, N1 (human), N1 (animal), N2, and N7, including typing of novel swine origin influenza A (H1N1) virus, during the 2009 outbreak in Milwaukee, Wisconsin. J. Clin. Microbiol. 47:2772-2778.

17. Hurt, A. C., et al. 2009. Performance of influenza rapid point-of-care tests in the detection of swine lineage $\mathrm{A}(\mathrm{H} 1 \mathrm{~N} 1)$ influenza viruses. Influenza Other Respi. Viruses 3:171-176.

18. Jiang, T., et al. 2010. Development of a real-time RT-PCR assay for a novel influenza A (H1N1) virus. J. Virol. Methods 163:470-473.

19. Kingsford, C., N. Nagarajan, and S. L. Salzberg. 2009. 2009 Swine-origin influenza A (H1N1) resembles previous influenza isolates. PLoS One 4:e6402.

20. Lalle, E., et al. 2010. Design and clinical application of a molecular method for detection and typing of the influenza A/H1N1pdm virus. J. Virol. Methods 163:486-488.

21. Lam, W. Y., et al. 2010. Development and comparison of molecular assays for the rapid detection of the pandemic influenza A (H1N1) 2009 virus. J. Med. Virol. 82:675-683.

22. Lu, Q., et al. 2009. Detection in 2009 of the swine origin influenza A (H1N1) virus by a subtyping microarray. J. Clin. Microbiol. 47:3060-3061.

23. Mackay, I. M., K. E. Arden, and A. Nitsche. 2002. Real-time PCR in virology. Nucleic Acids Res. 30:1292-1305.

24. Meijer, A., et al. 2009. Preparing the outbreak assistance laboratory network in the Netherlands for the detection of the influenza virus $\mathrm{A}(\mathrm{H} 1 \mathrm{~N} 1)$ variant J. Clin. Virol. 45:179-184.

25. Mitka, M. 2010. H1N1 influenza virus reassorting in pigs, poses unknown risk to human health. JAMA 304:626-627.

26. Ninove, L., et al. 2010. A simple method for molecular detection of swineorigin and human-origin influenza a virus. Vector Borne Zoonotic Dis. 10:237-240.

27. Pabbaraju, K., et al. 2009. Design and validation of real-time reverse transcription-PCR assays for detection of pandemic (H1N1) 2009 virus. J. Clin. Microbiol. 47:3454-3460.

28. Proudnikov, D., et al. 2003. Optimizing primer-probe design for fluorescen PCR. J. Neurosci. Methods 123:31-45.

29. Ren, L., et al. 2009. Prevalence of human respiratory viruses in adults with acute respiratory tract infections in Beijing, 2005-2007. Clin. Microbiol. Infect. 15:1146-1153.

30. Rozen, S., and H. Skaletsky. 2000. Primer3 on the WWW for general users and for biologist programmers. Methods Mol. Biol. 132:365-386.

31. Wang, R., Z. M. Sheng, and J. K. Taubenberger. 2009. Detection of novel (swine origin) H1N1 influenza A virus by quantitative real-time reverse transcription-PCR. J. Clin. Microbiol. 47:2675-2677.

32. Whiley, D. M., et al. 2009. Detection of novel influenza A(H1N1) virus by real-time RT-PCR. J. Clin. Virol. 45:203-204.

33. Yang, Y., et al. 2010. Simultaneous typing and HA/NA subtyping of influenza $A$ and $B$ viruses including the pandemic influenza A/H1N1 2009 by multiplex real-time RT-PCR. J. Virol. Methods 167:37-44. 